\title{
Caractéristiques Structurales Des Peuplements Ligneux À Syzygium Guineense (Willd.) DC. Subsp. Macrocarpum (Myrtaceae) En Vue De Leur Aménagement À Des Fins Apicoles Au Bénin
}

\author{
Badou Badjrêhou Romaël \\ Yedomonhan Hounnankpon \\ Laboratoire de Botanique et Ecologie Végétale, Département de Biologie \\ Végétale, Faculté des Sciences et Techniques, Université d'Abomey-Calavi \\ (UAC), Cotonou (Bénin)
}

doi: 10.19044/esj.2017.v13n24p186 URL:http://dx.doi.org/10.19044/esj.2017.v13n24p186

\begin{abstract}
The potential of honey production by honeybee in one area are determined by the flora. This study aims to evaluate the structural characteristics of Syzygium guineense subsp. macrocarpum so as to propose its promotion for beekeeping in Benin. Some structural variables were collected through 18 plots of one hectare $(100 \mathrm{~m} \times 100 \mathrm{~m})$. It was installed at random in three classified forests in sudano-guinean zone. Each plot was divided into 16 plots of $625 \mathrm{~m}^{2}(25 \mathrm{~m} \times 25 \mathrm{~m})$. For all species of tree and shrub, the stems of $\mathrm{dbh} \geq 10 \mathrm{~cm}$ have the highest values of density (549 stems/ha) and landed surface $\left(14.06 \mathrm{~m}^{2} / \mathrm{ha}\right)$ in dry dense forest. However, their middle height $(17.53 \mathrm{~m})$ and their middle diameter $(22.96 \mathrm{~cm})$ are more raised in wood land savanna. In regards to exclusive stems of $S$. guineense subsp. macrocarpum, their high values of density (6 stems/ha), landed surface $\left(0.30 \mathrm{~m}^{2} / \mathrm{ha}\right)$, and middle diameter $(17.84 \mathrm{~cm})$ were recorded in the dry dense forest. The relative contribution of $S$. guineense subsp. macrocarpum to woody population density varied between 0.45 and $1.04 \%$ for wood or shrub of $\mathrm{dbh} \geq 10 \mathrm{~cm}$. It also varies between 0.16 and $0.28 \%$ for wood or shrub of dbh< $10 \mathrm{~cm}$. The abundance index ranged from 0.09 to 0.77 and from 0.21 to 0.39 respectively for individual of $\mathrm{dbh} \geq 10 \mathrm{~cm} \mathrm{dbh}$ and $<10 \mathrm{~cm}$. The weak values of seedling density require an assessment of its fructiferous production.
\end{abstract}

Keywords: Syzygium guineense, structure, availability, Benin 


\section{Résumé}

Les potentialités de production de miel d'une zone de butinage des abeilles sont déterminées par son environnement floristique. L’objectif de cette étude est d'évaluer la structure de S. guineense subsp. macrocarpum en vue de son aménagement à des fins apicoles au Bénin. Des variables structurales ont été collectées à travers 18 placeaux d'un hectare $(100 \mathrm{~m} \mathrm{x}$ $100 \mathrm{~m}$ ), installés au hasard dans 3 forêts classées en zone soudanoguinéenne. Chaque placeau a été subdivisé en 16 placettes de $625 \mathrm{~m}^{2}(25 \mathrm{~m} \mathrm{x}$ $25 \mathrm{~m}$ ). En considérant l'ensemble des ligneux, les individus de $\mathrm{dbh} \geq 10 \mathrm{~cm}$ ont les valeurs les plus élevées de la densité (549 tiges/ha) en forêt dense sèche alors que leur hauteur moyenne $(17,53 \mathrm{~m})$ et leur diamètre moyen $(22,96 \mathrm{~cm})$ sont plus élevés au niveau de la forêt claire. En tenant compte exclusivement des individus de $S$. guineense subsp. macrocarpum, leurs valeurs élevées de densité (6 tiges/ha) et de diamètre moyen $(17,84 \mathrm{~cm})$ sont enregistrées en forêt dense sèche. La contribution relative de la sous-espèce à la densité des peuplements ligneux est comprise entre 0,45 et $1,04 \%$ pour les ligneux de $\mathrm{dbh} \geq 10 \mathrm{~cm}$ et entre 0,16 et $0,28 \%$ pour les ligneux de $\mathrm{dbh}<10$ $\mathrm{cm}$. L'indice d'abondance de $S$. guineense subsp. macrocarpum varie de 0,09 à 0,77 puis de 0,21 à 0,39 respectivement pour les individus de $\mathrm{dbh} \geq 10 \mathrm{~cm}$ et de $\mathrm{dbh}<10 \mathrm{~cm}$. Les faibles valeurs de densité des plantules nécessitent une évaluation de sa production fructifère.

Mots-clés : Syzygium guineense, structure, disponibilité, Bénin.

\section{Introduction}

L'apiculture est une activité génératrice de revenus et contribue aussi à l'amélioration des rendements agricoles par le biais de la pollinisation grâce aux abeilles (Vacher, 2007). Le miel doit sa valeur alimentaire et économique aux plantes. Il présente dans sa composition, son arôme, son goût, sa consistance et sa couleur, une gamme considérable de variations résultant, soit de l'identité de la plante sur laquelle le nectar a été récolté, soit des particularités régionales de la flore qui a offert asile aux butineuses (Louveaux, 1990). Les stratégies de butinage des abeilles assurent une grande efficacité pour la colonie car la majorité des butineuses est dirigée vers les endroits les plus rentables et avec une rapidité permettant l'exploitation de ressources éphémères (Waddington et al., 1994). L'attractivité d'un site mellifère dépend directement du gain énergétique qu'une butineuse peut en tirer par rapport aux dépenses d'énergie qu'elle devra faire lors de la manipulation des fleurs, de ses vols de fleurs en fleurs et de son retour, chargée, à la ruche (Moritz \& Southwick, 1992 ; Waddington et al., 1994). Les potentialités de production de miel d'une 
région donnée ou d'une zone de butinage sont déterminées par son paysage (Janssens et al., 2006).

Au Bénin, l'accumulation du miel dans les ruches coïncide avec la floraison de 9 espèces végétales en zone de transition soudano-guinéenne. Parmi ces dernières, Syzygium guineense (Willd.) DC. est l'espèce dont la production de nectar est largement élevée $(7,70-7,75 \mu 1 /$ fleur/heure au cours des heures de forte sécrétion nectarifère) (Yédomonhan, 2009). Des peuplements de $S$. guineense offriraient alors un rendement en miel élevé des ruches. Malheureusement, les travaux d'inventaires forestiers réalisés au Bénin indiquent que la densité de $S$. guineense au sein des peuplements ligneux est en moyenne de 0,31 tige/ha avec une régénération de 5 tiges/ha (MEPN, 2008). Cette densité moyenne au plan national de l'espèce est même nettement inférieure à celle de Afzelia africana (1,27 tige/ha) qui est pourtant considérée comme une espèce en danger au Bénin (Adomou, 2005) et vulnérable sur la liste rouge de l'UICN (2000). Il est donc nécessaire de connaître les caractérisations structurales de $S$. guineense dans ses habitats étant donné que les résultats d'inventaire national de MEPN (2008) ont été globalisants. En effet, il ne serait pas judicieux d'entreprendre des aménagements apicoles des communautés végétales abritant cette espèce à haute valeur mellifère en se basant exclusivement sur les résultats rapportés par l'inventaire forestier national dont l'échantillonnage paraît très grossier. De plus, selon Adjonou et al. (2016), les caractéristiques structurales des forêts représentent les indicateurs écologiques de leur aménagement.

Cette étude a donc pour but d'évaluer les caractéristiques structurales des peuplements ligneux à $S$. guineense subsp. macrocarpum à travers les différentes formations végétales afin d'entreprendre des stratégies d'aménagement de ces formations végétales à des fins apicoles.

\section{Méthodes}

\section{Site d'étude}

Le milieu d'étude correspond à la zone de transition guinéosoudanienne qui est subdivisé en trois secteurs phytogéographiques dont le secteur central sud à climax de type forêt dense sèche, le secteur central nord à climax de type miombo et le secteur central nord ouest qui comporte des îlots de forêt dense humide semi-décidue sèche (Akoègninou et al., 2006) (Figure 1). Le climat est de type tropical humide de transition avec une pluviosité annuelle de 1200 à $1300 \mathrm{~mm}$ d'eau. Les sols sont du type ferrugineux et ferralitique. La végétation est une mosaïque de forêt claire, de forêt dense sèche, de forêt dense humide semi-décidue, de savanes arborée et arbustive et de galerie forestière. L'agriculture est la principale activité réalisée dans le milieu d'étude. 


\section{Collecte de Données}

Afin de connaître la structure naturelle de S. guineense subsp. macrocarpum, trois (03) forêts classées ont été retenues dans la zone. Ces dernières ont été choisies de façon à avoir une forêt classée à l'ouest, une autre à l'est et une dernière au nord de la zone d'étude; le sud étant dépourvu de forêt classée. Il s'agit des forêts classées de Bassila à l'ouest, de Tchaourou à l'est et de N'Dali au nord de la zone d'étude (Figure 1). Au total, dix-huit (18) placeaux d'un hectare $(100 \mathrm{~m}$ x $100 \mathrm{~m})$ ont été installés au hasard, dont cinq (05) dans la forêt classée de Bassila, sept (07) à Tchaourou et six (06) à N'Dali. Chaque placeau a été subdivisé en seize (16) placettes de $625 \mathrm{~m}^{2}(25 \mathrm{~m} \mathrm{x} 25 \mathrm{~m})$ pour les relevés floristiques et forestiers.

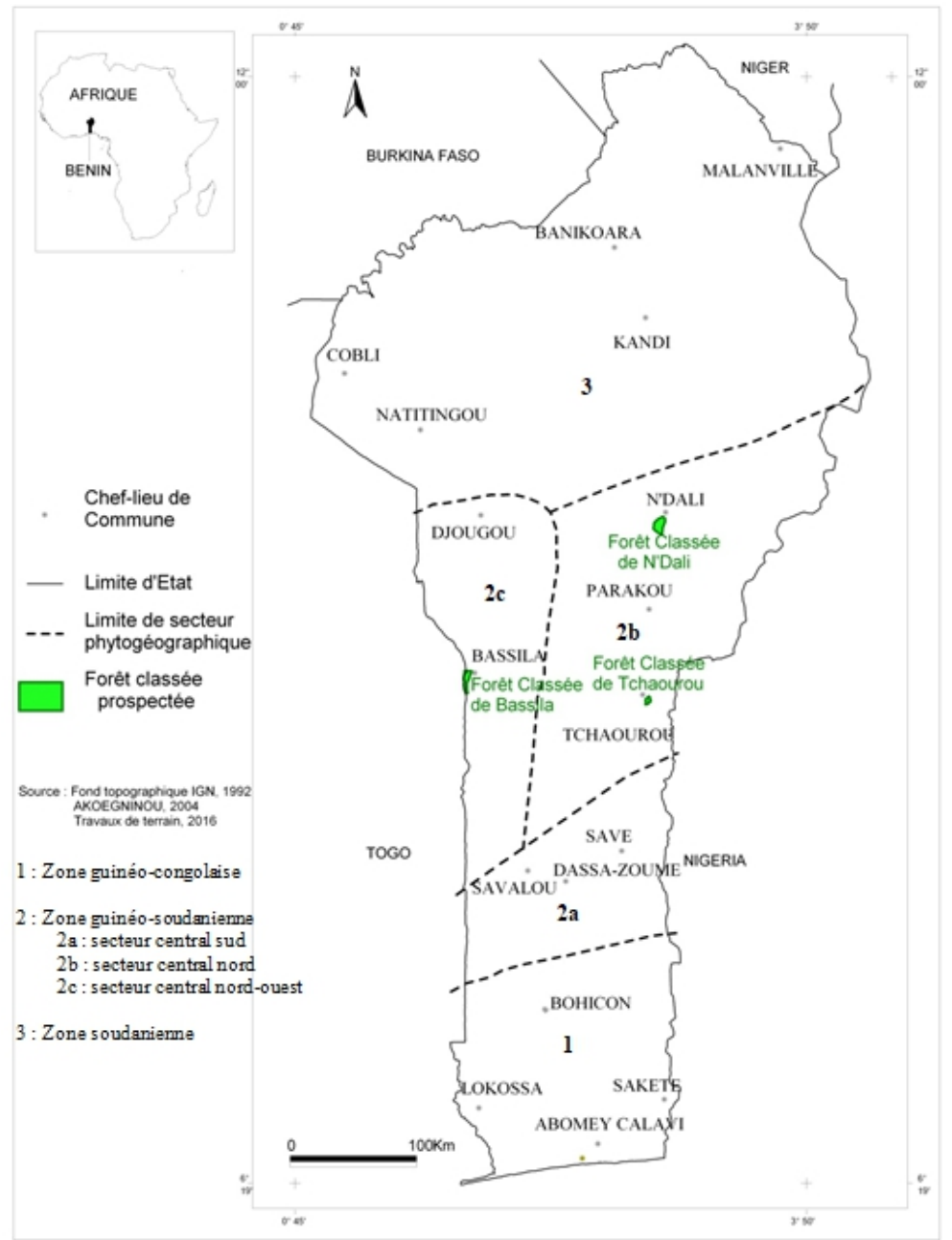

Figure 1. Localisation géographique des 3 forêts classées explorées en zone soudanoguinéenne au Bénin 
Les relevés floristiques ont consisté à noter la formation végétale et enregistrer toutes les espèces ligneuses. En ce qui concerne les relevés forestiers, la hauteur et le diamètre à hauteur de poitrine d'homme (dbh) ont été mesurés, respectivement avec le clinomètre Suunto et le ruban $\pi$, pour tous les arbres et arbustes. A cet effet, deux catégories d'individus ont été considérés, à savoir : la catégorie des individus ayant un $\mathrm{dbh}<10 \mathrm{~cm}$ et celle des individus à $\mathrm{dbh} \geq 10 \mathrm{~cm}$. Par ailleurs, les plantules de $S$. guineense subsp. macrocarpum ont été aussi comptées par placette.

\section{Traitement de Données}

La liste exhaustive des espèces ligneuses a été établie suivant la nomenclature botanique d'Akoègninou et al. (2006) et de Maroyi (2008). Les paramètres structuraux des peuplements ligneux (densité, surface terrière, diamètre moyen et hauteur moyenne) ont été évalués par forêt classée et par catégorie de ligneux (ligneux de $\mathrm{dbh}<10 \mathrm{~cm}$ et ligneux de $\mathrm{dbh}$ $\geq 10 \mathrm{~cm}$ ). Ils ont été déterminés en considérant l'ensemble des ligneux d'une part et Syzygium guineense subsp. macrocarpum seule d'autre part.

La densité $(\mathrm{N})$ des peuplements est le nombre de tiges par hectare. La surface terrière d'un peuplement est la somme des surfaces terrières de tous les arbres et arbustes qui constituent le peuplement. Elle est obtenue par la formule: $\mathrm{G}=(10000 \pi / 4 \mathrm{~s}) \sum\left(\mathrm{dbh}_{\mathrm{i}}{ }^{2}\right)$, avec $\mathrm{G}=$ surface terrière exprimée en $\mathrm{m}^{2} / \mathrm{ha} ; \mathrm{dbh}_{\mathrm{i}}=$ diamètre à hauteur de poitrine d'homme des arbres et arbustes en $\mathrm{m}$, et $\mathrm{s}=$ surface de relevé $\left(625 \mathrm{~m}^{2}\right)$.

La contribution de $S$. guineense subsp. macrocarpum à la structure de ses habitats a été évaluée à l'aide de 3 variables (la densité relative, la dominance relative et la présence relative), exprimées toutes en pourcentage. Par exemple, la densité relative de $S$. guineense subsp. macrocarpum est le rapport de sa densité et de celle de toutes les espèces ligneuses du milieu.

La disponibilité de $S$. guineense subsp. macrocarpum a été aussi évaluée par le biais de l'indice de rareté (IR) de Koné et al. (2013) et l'indice d'abondance. L'indice de rareté (IR) est donné par la formule : IR = [1$(\mathrm{n} / \mathrm{N})]^{*} 100$, avec $\mathrm{n}$ le nombre de placettes de $25 \mathrm{~m}$ x $25 \mathrm{~m}$ abritant l'espèce et $\mathrm{N}$ le nombre total de placettes pour le site. D'après Ferrari et al. (2000), l'espèce est dite commune si $\mathrm{RI}<0,20$ et rare si $\mathrm{RI}>0,20$.

L'indice d'abondance (Ia) a permis de comparer la densité de $S$. guineense subsp. macrocarpum à la densité moyenne/espèce ligneuse de chaque site afin de comparer son abondance par rapport aux autres espèces. Il a été déterminé suivant la formule :Ia : [Ds / (D/R)], où Ds est la densité de S. guineense subsp. macrocarpum, D est la densité moyenne obtenue par site pour l'ensemble des espèces ligneuses et $\mathrm{R}$ est le nombre d'espèces ligneuses recensées. L'espèce est dite faiblement abondante si Ia $<1$, moyennement abondante si $\mathrm{Ia}=1$ et abondante si $\mathrm{Ia}>1$. 
La relation entre le diamètre et la hauteur des individus de $S$. guineense subsp. macrocarpum a été déterminée. Les tests d'égalité de moyennes et de proportions ont été réalisés avec le logiciel Minitab pour comparer 2 à 2 les valeurs moyennes et les proportions des paramètres structuraux des 3 sites afin de voir si ces sites ont les mêmes aptitudes à l'apiculture.

\section{Résultats}

\section{Caractéristiques floristiques des peuplements ligneux à S. guineense subsp. macrocarpum}

Sur le plan physionomique, les zones d'inventaires sont constituées de forêt dense sèche à Bassila et de forêt claire au niveau des deux autres sites (Tchaourou et N'Dali). Au sein de ces peuplements ligneux, les espèces à forte fréquence de présence sont Isoberlinia doka (93-100\%) et Annona senegalensis (87-100\%) dans les forêts classées de Tchaourou et de N'Dali puis Terminalia avicennioides (100\%) et Vitellaria paradoxa $(94 \%)$ dans la forêt classée de Bassila.

$\mathrm{Au}$ total, 68 espèces d'arbres et d'arbustes ont été recensées dont 43 dans la forêt classée de Bassila (FCB), 51 dans la forêt classée de Tchaourou (FCT) et 54 dans la forêt classée de N'Dali (FCN) (Tableau 1). Les ligneux de $\mathrm{dbh}<10 \mathrm{~cm}$ sont partout plus diversifiés que ceux de $\mathrm{dbh} \geq 10 \mathrm{~cm}$.

L'indice de similarité de Jaccard indique une ressemblance floristique à degrés divers entre les trois sites. Il est de 0,50 entre la FCB et la FCT, de 0,58 entre la FCB et la FCN puis de 0,75 entre la FCT et la FCN. Ceci traduit une ressemblance floristique plus élevée entre les deux sites abritant le même type de formation végétale qui est la forêt claire.

Tableau 1. Richesse spécifique des sites

\begin{tabular}{ccccc}
\hline Catégories de ligneux & FCB & FCT & FCN & Zone d'étude \\
\hline $\begin{array}{c}\text { Arbres et arbustes de dbh } \geq 10 \\
\text { cm }\end{array}$ & 29 & 35 & 28 & 49 \\
$\begin{array}{c}\text { Arbres et arbustes de dbh<10 } \\
\text { cm } \\
\text { Total }\end{array}$ & 37 & 49 & 54 & 65 \\
\hline
\end{tabular}

FCB : forêt classée de Bassila, FCT : forêt classée de Tchaourou, FCN : forêt classée de

N'Dali

\section{Caractéristiques structurales des peuplements ligneux à S. guineense subsp. macrocarpum}

Les caractéristiques structurales des trois peuplements ligneux sont consignées dans le Tableau 2. En considérant les ligneux de $\mathrm{dbh} \geq 10 \mathrm{~cm}$, la forêt classée de Bassila enregistre la plus forte densité (549 tiges/ha), mais avec des individus de hauteur plus faible (11,49 $\mathrm{m}$ en moyenne). Par contre, la forêt classée de Tchaourou renferme les individus les plus gros $(22,96 \mathrm{~cm}$ 
de diamètre en moyenne) et les plus géants $(17,53 \mathrm{~cm}$ de hauteur en moyenne).

En tenant compte des arbres et arbustes de dbh $<10 \mathrm{~cm}$, c'est plutôt la forêt classée de N'Dali qui compte le plus grand nombre d'individus (1567 tiges/ha), avec la hauteur moyennela plus élevée $(5,07 \mathrm{~m})$. Mais, le diamètre moyen le plus élevé est plutôt observé dans la forêt classée de Bassila $(6,12$ $\mathrm{cm})$.

Les trois forêts classées, considérées deux à deux, enregistrent des valeurs moyennes parfois statistiquement différentes $(\mathrm{p}<0,05)$ et parfois similaires $(\mathrm{p}>0,05)$ pour les variables structurales. Par exemple, les forêts classées de Tchaourou et de N'Dali ont des valeurs similaires de densité et de hauteur pour les arbres de $\mathrm{dbh} \geq 10 \mathrm{~cm}$, mais différentes de celles obtenues dans la forêt classée de Bassila. De plus, la densité moyenne des ligneux de $\mathrm{dbh}<10 \mathrm{~cm}$ de la forêt classée de N'Dali est statistiquement différente et plus élevée que celle obtenue dans les deux autres sites qui ont des valeurs presque identiques.

Dans la forêt classée de Bassila, Vitellaria paradoxa est 1'espèce la plus abondante avec sa contribution de $22,49 \%$ et $19,05 \%$ respectivement pour les ligneux de $\mathrm{dbh} \geq 10 \mathrm{~cm}$ et ceux $\mathrm{de} \mathrm{dbh}<10 \mathrm{~cm}$. Elle est suivie de Terminalia avicennioides qui représente 17,49\% de 1'ensemble des arbres et arbustes de $\mathrm{dbh} \geq 10 \mathrm{~cm}$ et $15,60 \%$ de ceux de $\mathrm{dbh}<10 \mathrm{~cm}$. Au niveau, de la forêt classée de Tchaourou, les espèces les plus importantes, de par leur densité relative, sont Isoberlinia doka (29,57\%) pour les ligneux de $\mathrm{dbh} \geq 10$ cm, Annona senegalensis (12,09\%) et Uapaca togoense (10,58\%) pour les ligneux de $\mathrm{dbh}<10 \mathrm{~cm}$. Enfin, Isoberlinia doka a aussi la plus forte contribution $(41,18 \%$ ) à la densité des ligneux de $\mathrm{dbh} \geq 10 \mathrm{~cm}$ dans la forêt classée de N'Dali où Pericopsis laxiflora (10,83\%) et Annona senegalensis $(10,57 \%)$ prédominent les autres ligneux.

Tableau 2. Variation des paramètres structuraux au sein des sites pour l'ensemble des ligneux

\begin{tabular}{cccccc}
\hline $\begin{array}{c}\text { Catégories } \\
\text { de ligneux }\end{array}$ & $\begin{array}{c}\text { Paramètres } \\
\text { Structuraux }\end{array}$ & FCB & FCT & FCN & $\begin{array}{c}\text { Zone } \\
\text { d'étude }\end{array}$ \\
\hline $\begin{array}{c}\text { Arbres et } \\
\text { arbustes de }\end{array}$ & $\begin{array}{c}\text { Densité (tiges/ha) } \\
\text { Surface terrière } \\
\mathrm{dbh} \geq 10\end{array}$ & $549 \pm 110^{\mathrm{a}}$ & $274 \pm 100^{\mathrm{b}}$ & $204 \pm 84^{\mathrm{b}}$ & $366 \pm 183$ \\
$\mathrm{~cm}$ & & $14,06 \pm$ & $10,99 \pm$ & $5,74 \pm$ & $10,82 \pm$ \\
& Hauteur moyenne $(\mathrm{m})$ & $11,49 \pm$ & $17,53 \pm$ & $16,71 \pm$ & $14,90 \pm$ \\
& & $1,55^{\mathrm{a}}$ & $3,54^{\mathrm{b}}$ & $2,02^{\mathrm{b}}$ & 0,70 \\
& Diamètre moyen $(\mathrm{cm})$ & $18,85 \pm$ & $22,96 \pm$ & $18,88 \pm$ & $20,23 \pm$ \\
& $2,45^{\mathrm{a}}$ & $5,33^{\mathrm{b}}$ & $3,00^{\mathrm{a}}$ & 4,17 \\
\hline Arbres et & \multirow{2}{*}{ Densité (tiges/ha) } & $900 \pm 327^{\mathrm{a}}$ & $848 \pm 283^{\mathrm{a}}$ & $1567 \pm$ & $1060 \pm$ \\
arbustes de & Surface terrière & $2,29 \pm$ & $1,84 \pm$ & $2,61 \pm$ & 441 \\
dbh< $10 \mathrm{~cm}$ & $\left(\mathrm{~m}^{2} / \mathrm{ha}\right)$ & $1,17^{\mathrm{a}}$ & $0,82^{\mathrm{a}}$ & $0,81^{\mathrm{a}}$ & $2,23 \pm 1$ \\
& & $4,40 \pm$ & $3,87 \pm$ & $5,07 \pm$ & $4,40 \pm$ \\
& Hauteur moyenne $(\mathrm{m})$ & $0,38^{\mathrm{a}}$ & $0,66^{\mathrm{b}}$ & $0,57^{\mathrm{c}}$ & 0,70
\end{tabular}


$\begin{array}{ccccc}\text { Diamètre moyen }(\mathrm{cm}) & 6,12 \pm & 5,17 \pm & 4,58 \pm & 5,39 \pm \\ & 0,45^{\mathrm{a}} & 0,81^{\mathrm{b}} & 0,54^{\mathrm{c}} & 0,88\end{array}$

FCB : forêt classée de Bassila, FCT : forêt classée de Tchaourou, FCN : forêt classée de N'Dali. Pour une même variable, les moyennes suivies de lettres différentes en exposant sont significativement différentes $(\mathrm{p}<0,05)$ d'un site à l'autre et celles portant les mêmes lettres sont similaires $(\mathrm{p}>0,05)$.

\section{Caractéristiques dendrométriques et disponibilité de Syzygium guineense subsp. macrocarpum au sein de ses habitats}

Pour les individus de $S$. guineense subsp. macrocarpum d'au moins $10 \mathrm{~cm}$ de diamètre, la zone d'étude a une densité moyenne de 3 tiges/ha, une surface terrière moyenne de $0,09 \mathrm{~m}^{2} / \mathrm{ha}$, une hauteur moyenne de $8,78 \mathrm{~m}$ et un diamètre moyen de $16,37 \mathrm{~m}$ (Tableau 3 ). Les valeurs de ces paramètres varient d'un site à l'autre. La forêt classée de Bassila enregistre les plus fortes valeurs des paramètres structuraux (densité de 6 tiges/ha, surface terrière de $0,30 \mathrm{~m}^{2} /$ ha et diamètre moyen de $17,84 \mathrm{~m}$ ) à l'exception de la hauteur qui est plus élevée dans la forêt classée de N'Dali $(12,25 \mathrm{~m}$ en moyenne).

Quant aux pieds de $S$. guineense subsp. macrocarpum de dbh $<10$ $\mathrm{cm}$, la densité moyenne est de 2 tiges/ha, la surface terrière moyenne de 0,02 $\mathrm{m}^{2} / \mathrm{ha}$, la hauteur moyenne de 4,66 $\mathrm{m}$ et le diamètre moyen de $5,92 \mathrm{~cm}$ pour la zone d'étude. La forêt classée de N'Dali enregistre la plus forte densité (3 tiges/ha)et la hauteur moyenne la plus élevée $(6,43 \mathrm{~m})$. Par contre, le plus fort diamètre moyen $(6,73 \mathrm{~cm})$ a été obtenu dans la forêt classée de Bassila.

En ce qui concerne les plantules de $S$. guineense subsp. macrocarpum, la densité varie de 1 plant/ha à 5 plants/ha, avec une moyenne de 3 plants/ha pour la zone d'étude.

Les variables structurales de la forêt classée de Bassila ont des moyennes statistiquement différentes $(\mathrm{p}<0,05)$ de celles des deux autres forêts dont les moyennes sont identiques à l'exception de 3 variables (densité, surface terrière et hauteur moyenne) pour les individus à $\mathrm{dbh}<10$ $\mathrm{cm}$. Ces 3 variables sont statistiquement égales $(\mathrm{p}>0,05)$ pour toutes les trois forêts. La hauteur moyenne des arbres et arbustes de dhb $\geq 10 \mathrm{~cm}$ présente une différence significative d'une forêt à l'autre.

Les valeurs relatives de densité de $S$. guineense subsp. macrocarpum, de sa surface terrière et de sa présence sont consignées dans le Tableau 3. La forêt classée de Bassila connaît les contributions les plus élevéespour les différentes variables considérées pour deux catégories d'individus (individus $\geq 10 \mathrm{~cm}$ et plantules) alors que la forêt classée de Tchaourou suivie de N'Dali présente les contributions les plus élevéespour les mêmes variables considérées pourune seule catégorie d'individus (individus de $\mathrm{dbh}<10 \mathrm{~cm}$ ). 
Tableau 3. Paramètres structuraux de $S$. guineense subsp. macrocarpum au sein des 3 sites

\begin{tabular}{|c|c|c|c|c|c|}
\hline $\begin{array}{l}\text { Catégories } \\
\text { de ligneux }\end{array}$ & $\begin{array}{l}\text { Paramètres } \\
\text { structuraux }\end{array}$ & FCB & FCT & $\mathbf{F C N}$ & $\begin{array}{c}\text { Zone } \\
\text { d'étude }\end{array}$ \\
\hline \multicolumn{6}{|c|}{ Valeurs moyenne absolues des paramètres } \\
\hline \multirow{4}{*}{$\begin{array}{c}\text { Arbres et } \\
\text { arbustes de } \\
\text { dbh } \geq 10 \\
\mathrm{~cm}\end{array}$} & Densité (tiges/ha) & $\begin{array}{l}5,68 \pm \\
31,54^{\mathrm{a}}\end{array}$ & $1,24 \pm 6,89^{\mathrm{b}}$ & $\begin{array}{l}0,96 \pm \\
3,96^{\mathrm{b}}\end{array}$ & $2,63 \pm 18$ \\
\hline & $\begin{array}{l}\text { Surface terrière } \\
\qquad\left(\mathrm{m}^{2} / \mathrm{ha}\right)\end{array}$ & $\begin{array}{c}0,30 \pm \\
0,68^{\mathrm{a}}\end{array}$ & $\begin{array}{c}0,01 \pm \\
0,09^{\mathrm{b}}\end{array}$ & $\begin{array}{c}0,01 \pm \\
0,07^{\mathrm{b}}\end{array}$ & $\begin{array}{c}0,09 \pm \\
0,39\end{array}$ \\
\hline & Hauteur moyenne (m) & $\begin{array}{c}8,67 \pm \\
1,49^{\mathrm{a}}\end{array}$ & $\begin{array}{l}5,98 \pm \\
2,21^{\mathrm{b}}\end{array}$ & $\begin{array}{c}12,25 \pm \\
3,41^{\mathrm{c}}\end{array}$ & $\begin{array}{c}8,78 \pm \\
2,38\end{array}$ \\
\hline & Diamètre moyen $(\mathrm{cm})$ & $\begin{array}{c}17,84 \pm \\
4,38^{\mathrm{a}}\end{array}$ & $\begin{array}{c}11,42 \pm \\
1,84^{\mathrm{b}}\end{array}$ & $\begin{array}{c}12,52 \pm \\
3,14^{\mathrm{b}}\end{array}$ & $\begin{array}{c}16,37 \pm \\
4,70\end{array}$ \\
\hline \multirow{5}{*}{$\begin{array}{c}\text { Arbres et } \\
\text { arbustes de } \\
\mathrm{dbh}<10 \mathrm{~cm}\end{array}$} & & $1,44 \pm$ & $2,36 \pm$ & $3,48 \pm$ & $2,43 \pm$ \\
\hline & Densite (tiges/ha) & $24,65^{\mathrm{a}}$ & $19,87^{\mathrm{a}}$ & $21,18^{\mathrm{a}}$ & 21,70 \\
\hline & $\begin{array}{c}\text { Surface terrière } \\
\left(\mathrm{m}^{2} / \mathrm{ha}\right)\end{array}$ & $\begin{array}{c}0,03 \pm \\
0,10^{\mathrm{a}}\end{array}$ & $\begin{array}{l}0,02 \pm \\
0,05^{\mathrm{a}}\end{array}$ & $\begin{array}{c}0,01 \pm \\
0,05^{\mathrm{a}}\end{array}$ & $\begin{array}{c}0,02 \pm \\
0,07\end{array}$ \\
\hline & Hauteur moyenne (m) & $\begin{array}{c}4,50 \pm \\
1,59^{\mathrm{a}}\end{array}$ & $\begin{array}{c}3,84 \pm \\
1,25^{\mathrm{a}}\end{array}$ & $\begin{array}{c}6,43 \pm \\
3,39^{\mathrm{a}}\end{array}$ & $\begin{array}{c}4,66 \pm \\
2,41\end{array}$ \\
\hline & Diamètre moyen $(\mathrm{cm})$ & $\begin{array}{c}6,73 \pm \\
1,74^{\mathrm{a}} \\
\end{array}$ & $\begin{array}{c}5,40 \pm \\
1,27^{\mathrm{b}} \\
\end{array}$ & $\begin{array}{l}5,32 \pm \\
1,92^{\mathrm{b}} \\
\end{array}$ & $\begin{array}{c}5,92 \pm \\
1,72 \\
\end{array}$ \\
\hline Plantules & Densité (tiges/ha) & $\begin{array}{c}, 33 \pm \\
7,81^{\mathrm{a}} \\
\end{array}$ & $\begin{array}{c}2,71 \pm \\
2,87^{\mathrm{b}} \\
\end{array}$ & $\begin{array}{l}1,33 \pm \\
1,21^{\mathrm{b}} \\
\end{array}$ & $\begin{array}{c}2,72 \pm \\
4,48 \\
\end{array}$ \\
\hline \multicolumn{6}{|c|}{ Valeurs relatives des paramètres } \\
\hline \multirow{3}{*}{$\begin{array}{c}\text { Individus } \\
\text { de } d b h \geq 10 \\
\mathrm{~cm}\end{array}$} & Densité relative (\%) & $1,04^{\mathrm{a}}$ & $0,45^{\mathrm{b}}$ & $0,47^{\mathrm{b}}$ & 0,72 \\
\hline & $\begin{array}{c}\text { Dominance relative } \\
(\%)\end{array}$ & 2,13 & 0,11 & 0,17 & 0,84 \\
\hline & Présence relative $(\%)$ & $22,50^{\mathrm{a}}$ & $2,68^{\mathrm{b}}$ & $3,13^{\mathrm{b}}$ & 8,33 \\
\hline \multirow{3}{*}{$\begin{array}{c}\text { Individus } \\
\text { de } \mathrm{dbh}<10 \\
\mathrm{~cm}\end{array}$} & Densité relative $(\%)$ & $0,16^{\mathrm{a}}$ & $0,28^{\mathrm{a}}$ & $0,22^{\mathrm{a}}$ & 0,23 \\
\hline & $\begin{array}{c}\text { Dominance relative } \\
(\%)\end{array}$ & 0,56 & 1,52 & 0,85 & 0,94 \\
\hline & Présence relative (\%) & $9,38^{\mathrm{a}}$ & $18,75^{\mathrm{b}}$ & $13,39^{\mathrm{c}}$ & 13,54 \\
\hline
\end{tabular}

FCB : forêt classée de Bassila, FCT : forêt classée de Tchaourou, FCN : forêt classée de N'Dali. Les valeurs ayant des lettres différentes en exposant sont différentes $(p<0,05)$ d'un site à l'autre et celles ayant les mêmes lettres sont similaires $(\mathrm{p}>0,05)$.

L'indice de rareté de $S$. guineense subsp. macrocarpum est de 0,83 pour le milieu d'étude (Tableau 4). Il est partout supérieur à 0,20 et dénote de la rareté de l'espèce dans chacun des trois sites et par conséquent dans le milieu d'étude. Aussi, les indices d'abondance sont-ils partout nettement inférieur à 1 ; ceci témoigne de la faible abondance de $S$. guineense subsp. macrocarpum par rapport aux autres espèces ligneuses. Seuls les individus de ladite espèce ayant un $\mathrm{dbh} \geq 10$ dans la classée de Bassila ont une abondance relativement proche de celle de la densité moyenne par espèce en considérant tous les ligneux. 
Tableau 4. Paramètres de représentativité structurale de $S$. guineense subsp. macrocarpum

\begin{tabular}{|c|c|c|c|c|c|}
\hline & Indices & FCB & FCT & FCN & $\begin{array}{c}\text { Zone } \\
\text { d'étude }\end{array}$ \\
\hline & ice de rareté & 0,80 & 0,86 & 0,83 & 0,83 \\
\hline \multirow{2}{*}{$\begin{array}{c}\text { Indice } \\
\text { d'abondance }\end{array}$} & $\begin{array}{c}\text { Individus de } \mathrm{dbh} \geq 10 \\
\mathrm{~cm}\end{array}$ & 0,77 & 0,13 & 0,09 & 0,63 \\
\hline & $\begin{array}{c}\text { Individus de } \mathrm{dbh}<10 \\
\mathrm{~cm}\end{array}$ & 0,39 & 0,40 & 0,21 & 0,45 \\
\hline
\end{tabular}

FCB : forêt classée de Bassila, FCT : forêt classée de Tchaourou, FCN : forêt classée de N'Dali

\section{Fréquence de distribution de la densité des ligneux par classe de diamètres}

La distribution par classe de diamètres des individus au niveau des trois sites pour l'ensemble des ligneux d'une part et pour S. guineense subsp. macrocarpum exclusivement d'autre part est présentée par les Figures 2 et 3.

La distribution par classe de diamètres de l'ensemble des arbres et arbustes est une distribution normale tronquée avec une allure polynomiale à Bassila et exponentielle avec une allure de "J-renversé" au niveau des deux autres forêts classées (Tchaourou et N'Dali) (Figure 2).Les classes de diamètres les plus représentées en individus sont celles de 5 à $10 \mathrm{~cm}$ à Bassila (36,32\%) et de 0 à $5 \mathrm{~cm}$ à Tchaourou (32,98\%) et à N'Dali $(64,31 \%)$.

Par contre, en considérant seulement $S$. guineense subsp. macrocarpum, la distribution par classe de diamètres des individus est partout normale tronquée et s'ajuste des fonctions polynomiales (Figure 3). Les plus fortes densités relatives sont obtenues au niveau des classes de 5 à $10 \mathrm{~cm}$ à Bassila $(31,86 \%)$ et à N'Dali $(50 \%)$. Mais, à Tchaourou, les classes de 0 à $5 \mathrm{~cm}$ et de 5 à $10 \mathrm{~cm}$ sont les plus représentées avec $43,10 \%$ chacune. Par ailleurs, la forêt classée de Bassila renferme une proportion non négligeable $(12,39 \%)$ d'individus à diamètres supérieurs à $20 \mathrm{~cm}$ qui sont absents au niveau des deux autres sites. Aussi, totalise-t-elle, en considérant les classes de diamètres supérieurs à $10 \mathrm{~cm}, 59,29 \%$ des tiges contre seulement $13,21 \%$ pour la forêt classée de Tchaourou et $10 \%$ pour la forêt classée de N'Dali. 

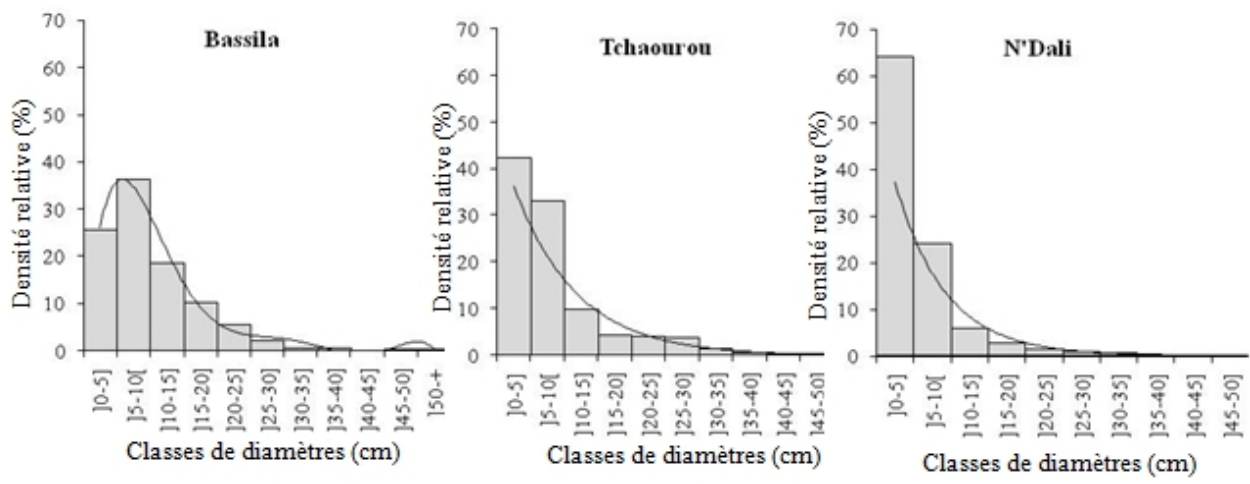

Figure 2. Fréquence de distribution par classe de diamètres des individus d'arbres et arbustes de toutes les espèces
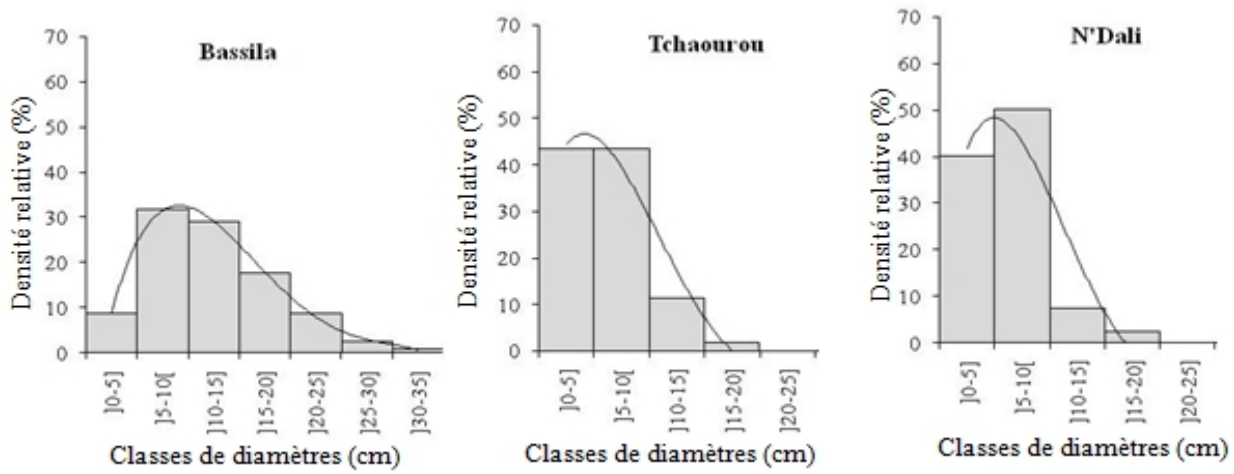

Figure 3. Fréquence de distribution par classe de diamètres des individus de Syzygium guineense subsp. macrocarpum seule

Relation entre le diamètre et la hauteur des arbres et arbustes de $S$. guineense subsp. macrocarpum

La forme des arbres et arbustes a été évaluée par le biais de la relation entre la hauteur et le diamètre des individus de la sous-espèce. La Figure 4 indique que la hauteur de Syzygium guineense subsp. macrocarpum peut être estimée, avec $72 \%$ de succès, à partir du diamètre des arbres et arbustes. Le meilleur ajustement entre la hauteur $(\mathrm{H})$ et le diamètre $(\mathrm{D})$ est une fonction puissance d'équation : $\mathrm{H}=0,963 \mathrm{D}^{0,77}$. 


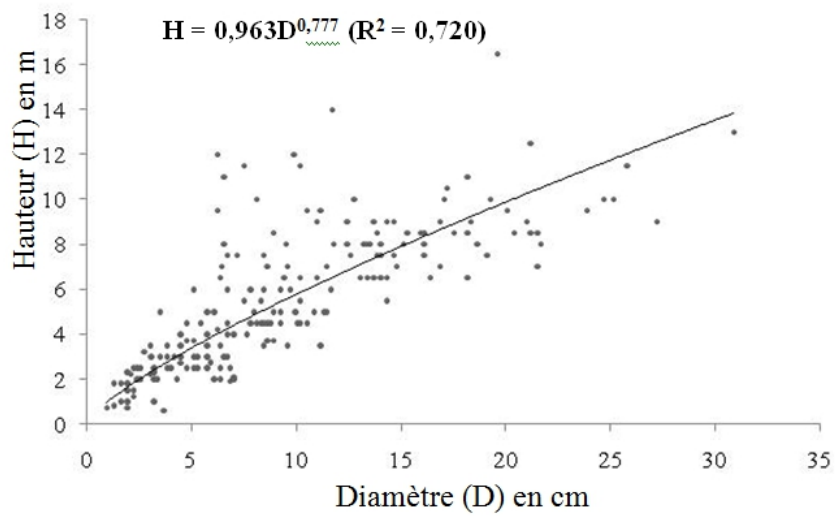

Figure 4. Ajustement de la relation entre la hauteur et le diamètre des individus de $S$. guineense subsp. macrocarpum

\section{Discussion}

Variabilité structurale des communautés végétales abritant $S$. guineense subsp. macrocarpum

L'analyse structurale des peuplements ligneux à S. guineense subsp. macrocarpum a été faite à l'aide d'inventaires floristiques et forestiers dans trois sites en zone phytogéographique soudano-guinéenne du Bénin. Parmi ces sites, deux sont similaires sur le plan physionomique (forêts classées de Tchaourou et de N'Dali) alors que le troisième diffère des deux premiers. Ces résultats s'expliquent par le fait que les forêts classées de Tchaourou (FCT) et de N'Dali (FCN) appartiennent au même secteur phytogéographique (le secteur central nord) alors que la forêt classée de Bassila (FCB) est du secteur central nord-ouest (Akoègninou et al., 2006). De plus, les zones d'inventaires sont occupées par la même formation végétale (forêt claire à Isoberlinia doka) dans les forêts classées de Tchaourou et de N'Dali. Par contre, à Bassila, il s'agit d'une autre formation végétale (forêt dense sèche à Vitellaria paradoxa).

Les caractéristiques structurales ont aussi mis en exergue la forte ressemblance des sites de Tchaourou et de N'Dali qui sont les moins structurées en termes de densité et de surface terrière comparativement au site de Bassila. Les valeurs moyennes de densité des peuplements ligneux de $\mathrm{dbh} \geq 10 \mathrm{~cm}$ obtenues dans les forêts claires à Isoberlinia doka (274 tiges/ha à Tchaourou et 204 tiges/ha à N'Dali) sont nettement inférieures à celles de 349-376 tiges/ha trouvées par Sokpon et al. (2006) pour les forêts claires à Isoberlinia doka du nord-Bénin. Ces écarts peuvent s'expliquer par le fait que la structure des communautés végétales peut varier d'un site à l'autre et d'une région à l'autre à cause de divers facteurs dont les conditions écologiques, l'état de la végétation, l'intensité de la pression humaine et anthropiques (Glèlè et al., 2008; Biresaw \& Pavliš, 2010; Feldpausch et al., 2011; Imani Magisho et al., 2016). Les valeurs de régénérations (ligneux de 
$\mathrm{dbh}<10 \mathrm{~cm})(848$ à 1547 tiges/ha) sont similaires à celles de 304 à 1466 tiges/ha signalées par Sokpon et al. (2006). Elles ne sont donc pas spécifiques aux peuplements ligneux étudiés mais constituent une caractéristique générale pour les forêts claires et forêts denses en zone de savane au Bénin. La distribution par classe de diamètre des arbres et arbustes présente une allure en "J-renversé" et traduit la forte abondance des individus à forte diamètre contre une faible abondance des individus à gros diamètre. Ce type de distribution n'est pas une particularité des sites étudiés mais il est propre aux forêts tropicales en dynamique progressive (Hitimana et al., 2004; Imani Mugisho et al., 2016). Il dénote de l'existence probable d'une pression anthropique centrée sur les individus de gros diamètres. Pour les peuplements de Isoberlinia spp., Glèlè \& Sinsin (2009) ont obtenu une distribution similaire en savane arborée.

\section{Caractéristiques structurales de $S$. guineense subsp. macrocarpum}

Les caractéristiques structurales (densité, surface terrière, hauteur, diamètre) des peuplements de Syzygium guineense subsp. macrocarpum ont montré des valeurs moyennes similaires entre les forêts classées de Tchaourou et de N'Dali où c'est le même type de formation végétale (forêt claire à Isoberlinia doka) prospecté. Par contre, les valeurs moyennes obtenues pour ces mêmes paramètres structuraux au niveau de la forêt dense sèche (site de Bassila) sont pour la plupart différentes et plus élevées que celles trouvées dans les deux autres sites. Cette meilleure structuration du peuplement de $S$. guineense subsp. macrocarpum à Bassila est confortée par la structure diamétrique de la sous-espèce qui suit une distribution normale avec une abondance relativement importante des individus à gros diamètres comparativement aux deux autres sites. La forte représentativité des individus de faibles diamètres à Tchaourou et à N'Dali est bien observée pour l'ensemble des ligneux et pour $S$. guineense subsp. macrocarpum seule. Au niveau de ces sites, la régénération est alors disponible et abondante mais les individus ne parviennent pas à atteindre leur phase maximale de développement. Cela dénote de l'existence probable aussi d'une pression anthropique centrée sur les individus de gros diamètres à l'image des autres espèces ligneuses.

L'indice d'abondance de $S$. guineense subsp. macrocarpum indique que cette dernière fait partie des espèces très peu représentées dans les habitats explorés surtout au niveau des forêts claires où il est nettement inférieur à $1(0,09$ à 0,13$)$. La densité obtenue qui n'est que de 6 tiges/ha en forêt dense sèche et de 0 à 1 tige/ha en forêt claire pour les ligneux de $\mathrm{dbh} \geq$ $10 \mathrm{~cm}$ est légèrement au-dessus de celle de 0,30 tige/ha trouvée au plan national lors du récent inventaire forestier national du Bénin (MEPN, 2008). La différence serait liée à la taille de l'échantillon et au fait que les travaux 
d'inventaire forestier national ont porté sur les habitats de prédilection ou non de l'espèce. L'indice de rareté obtenu $(0,80-0,86)$ témoigne de ce que $S$. guineense subsp. macrocarpum peut être classée parmi les espèces rares pour la zone d'étude. Il est proche de celui de 0,84 obtenu pour Afzelia africana et de 0,86 pour Milicia excelsa qui sont toutes classées dans la catégorie des espèces en danger au Bénin par Adomou (2005). De plus, le cumul des densités des trois catégories d'individus (individus de $\mathrm{dbh} \geq 10 \mathrm{~cm}$, individus de $\mathrm{dbh}<10 \mathrm{~cm}$ et les plantules) est de 8 tiges/ha pour la zone d'étude et varie de 5 à 12 tiges/ha selon le site. Cette densité cumulée de $S$. guineense subsp. macrocarpum reste partout nettement inférieure à celle attendue étant donné que la production florale de l'espèce a été évaluée à 45 fleurs/inflorescence et 57746 fleurs/arbre par Yédomonhan (2009). Ceci met en exergue la nécessité d'évaluer la production fructifère de l'espèce afin de vérifier si sa très faible densité n'est pas liée à la faible disponibilité de semences.

\section{Potentialités mellifères des habitats explorés et leurs implications pour la production apicole}

Sur le plan floristique, la flore ligneuse des 3 forêts classées étudiées, riche de 68 espèces, renferme 44 espèces (soit 64,71\%) mellifères dont $43,18 \%$ sont à la fois nectarifères et pollinifères, 40,91\% sont exclusivement nectarifères et 15,91\% sont exclusivement pollinifères d'après les travaux de Yédomonhan et al. (2009) en zone soudano-guinéenne au Bénin. Aucune des 24 espèces restantes n'a été reconnue mellifère par les divers travaux en apiculture (Nombré, 2003 ; Dongock Nguemo et al., 2004; Yédomonhan, 2009; Iritié et al., 2014). Les 3 communautés végétales étudiées ne détiennent donc pas des atouts floristiques par rapport à ceux rapportés par Yédomonhan et al. (2009). Au contraire, elles ne renferment que 3 ( $S$. guineense, Burkea africana et Terminalia avicennoides) des 9 espèces identifiées comme plantes à haute valeur mellifère par les mêmes auteurs. Les 3 forêts classées étudiées n'ont donc pas les potentialités floristiques nécessaires pour induire une production intensive de miel. Ceci confirme alors les observations de Iritié et al. (2014) qui ont affirmé que la production $\mathrm{du}$ miel dépend du potentiel mellifère individuel des espèces végétales localisées dans les ruchers.

Les caractéristiques structurales de $S$. guineense subsp. macrocarpum obtenues traduisent d'une part l'inégalité des potentialités apicoles au sein des 3 forêts classées étudiées et d'autre part la faible aptitude apicole de ces sites. En comparant les 3 sites, la forte représentativité des individus à gros diamètres dans la forêt classée de Bassila prédispose mieux ce site à l'activité apicole contrairement aux autres sites où les individus matures sont peu représentés. Cependant en faisant référence à la disponibilité de l'espèce 
par rapport aux autres espèces ligneuses des communautés végétales prospectées, la faible disponibilité de S. guineense subsp. macrocarpum constitue des limites de ces communautés végétales pour une production apicole accrue et rentable. Ceci serait à l'origine des faibles rendements en miel obtenus (10,55 à 11,2 litres de miel/ruche) au Bénin dans des habitats où $S$. guineense subsp. macrocarpum est présente (Yédomonhan et al., 2009; Ahouandjinou et al., 2016). La rentabilité apicole des sites étudiés pourraient donc passer par leur enrichissement en $S$. guineense subsp. macrocarpum reconnue comme espèce à forte source de nectar pour les abeilles (Arbonnier, 2008; Maroyi, 2008; Yédomonhan, 2009; Djonwangwe et al., 2011).

\section{Conclusion}

Cette étude a permis de recenser la flore ligneuse et de décrire la structure des formations végétales abritant $S$. guineense subsp. macrocarpum. Elle a permis aussi de savoir que les caractéristiques structurales de la sous-espèce dépendent de la nature de la formation végétale et présentent une meilleure structuration en forêt dense sèche. Les structures diamétriques des ligneux dans les 3 sites mettent en évidence l'existence d'une pression anthropique sur les individus matures au niveau des forêts classées de Tchaourou et de N'Dali. La flore ligneuse recensée renferme une gamme assez variée d'espèces mellifères mais qui sont de faible valeur mellifère, à l'exception de 3 espèces dont Syzygium guineense. Malheureusement, S. guineense est une espèce à abondance relativement faible à très faible. Sa disponibilité n'offrirait pas une activité apicole accrue et rentable au niveau des communautés végétales qui l'abritent. Compte tenu de sa forte valeur mellifère, il serait judicieux de l'utiliser pour enrichir les sites apicoles pour améliorer leur rendement en miel et promouvoir l'apiculture. Par ailleurs, la faible densité de l'espèce suscite des questions de recherche relatives à sa production fructifère.

\section{Remerciements}

Les auteurs remercient très sincèrement la Fondation Internationale pour la Science (FIS) pour avoir financé cette recherche à travers la bourse D/4014-2 octroyée au co-auteur de cet article.

\section{References:}

1. Adjonou, K., Radji, A. R., Kokutse, A. D., \& Kokou, K. (2016). Considération des caractéristiques structurales comme indicateurs écologiques d'aménagement forestier au Togo (Afrique de l'Ouest). VertigO - la revue électronique en sciences de l'environnement. Volume 16 Numéro 1. 
2. Adomou, A. C. (2005). Vegetation patterns and environmental gradient in Benin: Implications for biogeography and conservation. PhD thesis, Wageningen University, the Netherlands, $136 \mathrm{p}$.

3. Ahouandjinou, T. B., Yédomonhan, H., Adomou, A. C., Tossou, M. G., \& Akoègninou, A. (2016). Caractéristiques techniques et importance socio-économique de l'apiculture au Nord-Ouest du Bénin : cas de la commune de Cobly. Int. J. Biol. Chem. Sci. 10 (3): 1350-1369.

4. Akoègninou, A., van der Burg, W. J., \& van der Maesen, L. J. G. (2006). Flore Analytique du Bénin. Backhuys Publishers, Wageningen, $1034 \mathrm{p}$.

5. Arbonnier, P. B. M. (2008). Ligneux du Sahel. Outil graphique d'identification V.1.0. CIRAD.

6. Biresaw, M. A., \& Pavliš, J. (2010). Vegetation structure and density of woody plant species in two woodland areas of Amhara National Regional State, Ethiopia. Acta Univ. Agric. Silvic. Mendelianae Brun., 58, 21-32.

7. Djonwangwe, D., Tchuenguem Fohouo, F.-N., Messi, J., \& Brückner, D. (2011). Foraging and pollination activities of Apis mellifera adansonii Latreille (Apidae) on Syzygium guineense var. guineense (Myrtaceae) flowers at Ngaoundéré (Cameroon) Journal of Animal \& Plant Sciences, 10 (3): 1325-1333.

8. Dongock Nguemo, D., Foko, J., Pinta, J.Y., Ngouo, L.V., Tchoumboue, J., \& Zango, P. (2004). Inventaire et identification des plantes mellifères de la zone soudano-guinéenne d'altitude de l'ouest Cameroun. Tropicultura 22 (3) 139-145.

9. Feldpausch, T.R., Banin, L., Phillips, O.L., Baker, T.R., Lewis, S.L., Quesada, C.A., Affum-Baffoe, K., Arets, E.J.M.M., Berry, N.J., Bird, M., Brondizio, E.S., de Camargo, P., Chave, J., Djagbletey, G., Domingues, T.F., Drescher, M., Fearnside, P.M., França, M.B., Fyllas, N.M., Lopez-Gonzalez, G., Hladik, A., Higuchi, N., Hunter, M.O., Iida, Y., Salim, K.A., Kassim, A.R., Keller, M., Kemp, J., King, D.A., Lovett, J.C., Marimon, B.S., et al. (2011). Heightdiameter allometry of tropical forest trees. Biogeosciences, 8, 10811106.

10. Ferrari C., Pezzi G., \& Portanova, A. (2000). The northern slope of Mount Prado : a diversity hotspot within northern Apennine summit vegetation. Proceeding IAVS Symposium. LAVS, Opulus Press Uppsala, Sweden, (pp. 198-202).

11. Glèlè Kakaï, R., \& Sinsin, B. (2009). Structural description of two Isoberlinia dominated vegetation types in the Wari-Maro Forest Reserve (Benin). South African Journal of Botany (75) (pp. 43-51). 
12. Glèlè Kakaï, R., Sinsin, B., \& Palm, R. (2008). Etude dendrométrique de Pterocarpus erinaceus Poir. Des formations naturelles de la zone soudanienne au Bénin. Agronomie africaine 20 (3) (pp. 245-255).

13. Hitimana, J., Kiyiapi, J.L., \& Njunge, J.T. (2004). Forest structure characteristics in disturbed and undisturbed sites of Mt. Elgon Moist Lower Montane Forest, western Kenya. For. Ecol. Manage., 194, 269-291.

14. Imani Mugisho, G., Zapfack, L., Riera, B., Mwanga Mwanga, I.J.-C., Bulonvu, F., \& Boyemba, F. (2016). Variabilité structurale des Peuplements d'arbres en forêt de montagne du Parc National de Kahuzi-Biega et ses environs, RD. Congo. European Scientific Journal. Edition vol.12, No.23 ISSN: $1857-7881$.

15. Iritié, B. M., Wandan, E. N., Paraiso A. A., Fantodji, A., \& Lohoué Gbomene, L. (2014). Identification des plantes mellifères de la zone agroforestière de l'Ecole Supérieure Agronomique de Yamoussoukro (Côte d'Ivoire). European Scientific Journal, édition vol.10, No.30 ISSN: 1857 - 7881 (Print) e - ISSN 1857- 7431.

16. Janssens, X., Bruneau, E., \& Lebrun, P. (2006). Prévision des potentialités de production de miel à l'échelle d'un rucher au moyen d'un système d'information géographique. Apidologie (37) (pp. 351$365)$.

17. Koné, M. W., Dro, B., Soro, D., Bakayoko, A., \& Kamanzi, K. (2013). Evaluation de l'abondance de plantes médicinales utilisées en médecine traditionnelle dans le Nord de la Côte d'Ivoire. Journal of Animal \& Plant Sciences 17 (3): 2631-2646.

18. Louveaux, J. (1990). Les relations abeilles-pollens, Bulletin de la Société Botanique de France. Actualités Botaniques, 137 (2), 121131.

19. Maroyi, A. (2008). Syzygium guineense (Willd.) DC. In Louppe, D., Oteng-Amoako, A.A. \& Brink, M. (Editors). Prota 7(1): Timbers/Bois d'œuvre 1. PROTA (Plant Resources of Tropical Africa / Ressources végétales de l'Afrique tropicale), Wageningen, Netherlands / Pays Bas.

20. MEPN (2008). Rapport d'excécution d'inventaire forestier au Bénin. Cotonou, Bénin, 121 p. + annexes.

21. Moritz, R. F. A., \& Southwick, E. E. (1992). Bees as superorganisms, An evolutionary reality, Springer Verlag, Berlin, Heidelberg.

22. Nombré I. (2003). Etudes des potentialités mellifères de deux zones du Burkina Faso: Garango (province du Bouglou) et Nazinga (province du Nahouri). Thèse de Doctorat d'Université, Université de Burkina Faso, 156 p.Sokpon, N., Biaou, S.H., Ouinsavi, C., \& 
Hunhyet, O. (2006). Bases techniques pour une gestion durable des forêts claires $\mathrm{du}$ nord-Bénin: rotation, diamètre minimal d'exploitabilité et régénération. Bois et forêts des Tropiques 287 (1) (pp. 45-57).

23. Vacher, R. (2007). L'apiculture à considérer à sa juste valeur en agriculture. Mémoire présenté lors de la Commission sur l'Avenir de l'Agriculture et de l'Agroalimentaire du Québec (CAAAQ)/ Fédération des Apiculteurs du Québec (FAQ). 16 p.

24. Waddington, K. D., Visscher, P. K., Herbert, T. J., \& Richter, M. R. (1994). Comparisons of forager distributions from matched honeybee colonies in suburban environments. Behav. Ecol. Sociobiol. (35) (pp. 423-429).

25. Yédomonhan, H. (2009). Plantes mellifères et potentialités de production de miel en zone guinéenne et soudano-guinéenne au Bénin. Thèse de Doctorat de l'Université d'Abomey-Calavi (Bénin), FAST/UAC, $273 \mathrm{p}$.

26. Yédomonhan, H., Tossou, M. G., \& Akoègninou, A., Demènou, B. B., \& Traoré, D. (2009). Diversité des plantes mellifères de la zone soudano-guinéenne: cas de l'arrondissement de Manigri (CentreOuest du Bénin). Int. J. Biol. Chem. Sci. 3(2): 355-366. 